ORIGINAL ARTICLE

\title{
Pulmonary and sinus diseases in primary humoral immunodeficiencies with chronic productive cough
}

\author{
F Rusconi, C Panisi, R M Dellepiane, F Cardinale, L Chini, B Martire, N Bonelli, G Felisati, \\ M C Pietrogrande
}

See end of article for authors' affiliations

....................

Correspondence to:

Dr F Rusconi, Department of Paediatrics, University of Milan, Via Commenda 9, 20122 Milan, Italy;

franca.rusconi@unimi.it

Accepted 13 May 2003

\begin{abstract}
Aims: To prospectively evaluate sinopulmonary disease in 24 patients with primary humoral immunodeficiency $(11$ with agammaglobulinaemia, nine with isolated $\lg \mathrm{A}$ deficiency, and two with common variable immunodeficiency) and chronic productive cough, ascertain the usefulness of chest high resolution computed tomography (HRCT) in evaluating the progression of lung disease, and test a therapeutic approach to chronic sinusitis.

Methods: Pulmonary abnormalities were evaluated using lung function tests and HRCT (Bhalla score); chronic sinusitis was diagnosed clinically and confirmed by flexible fibreoptic endoscopy. Sixteen patients entered the three year follow up.

Results: Lung function testing revealed an obstruction in four patients; chest HRCT was abnormal in 16. There was a linear relation between the Bhalla score $\geqslant 4$ and the number of months with cough/year over the previous two years (clinical score), and between the difference in clinical scores during follow up and in the previous two years and the difference in Bhalla score. The pulmonary lesions did not significantly progress over a three year period. Thirteen patients had chronic sinusitis; $6 / 10$ patients followed up were successfully treated with antibiotics plus topical therapy and two with nasal polypoid disease with endoscopic sinus surgery.

Conclusions: In patients with primary humoral immunodeficiency and chronic productive cough, HRCT is very useful in delineating the extent of lung damage. The correlation between Bhalla score and clinical findings and the favourable outcome of the disease suggests that in most patients chest HRCT should not be repeated annually as previously suggested. Medical therapy seems to be effective in many cases of chronic sinusitis.
\end{abstract}

$\mathrm{T}$ he availability of broad spectrum antibiotics, together with intravenous immunoglobulin infusions to keep serum IgG concentrations within physiological levels, ${ }^{1-3}$ has significantly reduced the frequency and severity of acute bacterial infections in patients with hypogammaglobulinaemia. As a consequence, there has been a reduction in the severe chronic sinopulmonary infections leading to bronchiectasis, atelectasis, pulmonary fibrosis, and eventually cor pulmonale and respiratory failure. ${ }^{4-7}$

This progress has been associated with the use of more sensitive diagnostic techniques such as high resolution computed tomography (HRCT) and nasal endoscopy.

Given the lack of recent prospective paediatric studies evaluating the development of sinopulmonary complications in patients with humoral immunodeficiencies, the primary aim of this prospective multicentre study was to evaluate lung and sinus diseases clinically, and by means of HRCT and nasal endoscopy, in patients with chronic productive cough affected by $\mathrm{X}$ linked and autosomal recessive agammaglobulinaemia (XLA, ARA), common variable immunodeficiency (CVDI), and isolated IgA deficiency (IgA-D). Although most IgA-D patients are asymptomatic or only have recurrent acute sinopulmonary infections in the first years of life, some may be affected by bronchiectasis.

The decision to evaluate patients with different humoral immunodeficiencies in a single study was based on the fact that all of the patients shared the common clinical presentation of chronic cough and phlegm (both of which are markers of chronic bronchitis and sinusitis), which in patients with hypogammaglobulinaemia may be related to the failure of immunoglobulin therapy to restore antibody concentrations on mucosal surfaces. ${ }^{8}$ On the other hand, limiting the study to only one disease would not have guaranteed any greater patient homogeneity because the results of genetic mutation analyses of XLA patients suggest that it is still not possible to make genotype/phenotype correlations, ${ }^{9}$ and that there is extreme variation in the phenotype expression of affected family members although they share the same genetic abnormality. ${ }^{10}$ Furthermore, genetic studies of families affected by both IgA-D and CVID suggest that these disorders may have a common basis. ${ }^{11}$ It therefore seemed to be clinically logical to use the same study protocol to follow the respiratory outcomes of patients with different defects but a very similar clinical presentation.

The further aims of the study were to ascertain the advantage of chest HRCT over clinical judgement in prospectively evaluating the progression of lung disease in patients thoroughly followed up in one centre, and to standardise a diagnostic and therapeutic approach to chronic sinusitis. Worsening HRCT findings despite adequate intravenous immunoglobulin therapy over a period of 3-5 years were reported in three of the children participating in a retrospective study of children with various primary antibody deficiency disorders, ${ }^{12}$ and in two children participating in a prospective study of children and adults with primary hypogammaglobulinaemia. ${ }^{13}$ The authors of both of these

Abbreviations: ARA, autosomal recessive agammaglobulinaemia; $\mathrm{CVDI}$, common variable immunodeficiency; $\mathrm{FEV}_{1}$, forced expiratory volume in one second; FVC, forced vital capacity; HRCT, high resolution computed tomography; VC, vital capacity; XLA, X linked agammaglobulinaemia 
studies advocated regular annual or bi-annual HRCT examinations in order to optimise pulmonary care.

\section{METHODS}

\section{Inclusion criteria and patient characteristics}

We studied 24 patients affected by primary humoral immunodeficiencies (16 males and eight females; age 2.719.8 years, median 11 years) attending the paediatric departments of the Universities of Milan (16 patients), Bari ( 5 patients), and Rome ( 3 patients) in 1998. The patients were included in the study if they had had cough and phlegm for at least three months/year in the previous two years. The Ethics Commission of the University of Milan approved the study protocol. The parents of one patient with XLA and chronic cough and phlegm refused chest HRCT, and so the patient was excluded from the study.

Eleven of the 24 patients were diagnosed as having XLA, two as having ARA, two as having CVID, and nine as having isolated IgA-D according to the diagnostic criteria of PAGID (the Pan-American Group for Immunodeficiency) and ESID (the European Society for Immunodeficiencies). ${ }^{14}$ The patients with XLA were all male with a CD19+ B cell count of less than $2 \%$ and a Btk mutation and/or no Btk mRNA at Northern blot analysis of neutrophils or monocytes. ARA was diagnosed in two sisters on the basis of CD19+ B cell counts of less than $2 \%$, serum IgG, IgA and IgM levels of more than 2 SD below the normal values for their age, recurrent bacterial infections in the first five years of life, and negative genetic examinations for mutations in Btk, $\operatorname{Ig} \alpha, \operatorname{Ig} \beta, \mu$ heavy chain, Vpre $\beta$, and $\lambda 5 / 14$.1. The diagnostic criteria for CVID were serum IgG, IgA, and/or IgM levels of 2 SD or more below the age related mean, the absence of isohaemagglutinins, and/or a poor response to vaccines. A diagnosis of IgA deficiency (IgA-D) was made in the presence of serum IgA levels of less than $7 \mathrm{mg} / \mathrm{dl}$, but normal $\operatorname{IgG}, \operatorname{IgM}$, and IgG antibody responses to vaccination.

\section{Baseline data collection}

The clinical records of the patients were reviewed and the following variables recorded: age at diagnosis, number of months with cough and phlegm over the previous two years, the lifetime number of pneumonia episodes confirmed by chest radiography, and, in the patients with XLA, ARA, and CVID, the length and type of immunoglobulin replacement therapy, and their age at the start of therapy.

\section{Evaluation of pulmonary and sinus disease at entry}

All of the patients underwent chest HRCT at least two months after an acute respiratory illness. The scans were performed using a standard high resolution technique with the patient's respiration held in deep inspiration. Sections with a thickness of $1-2 \mathrm{~mm}$ were obtained at $8-10 \mathrm{~mm}$ intervals and reconstructed using a bone algorithm. All of the scans were reviewed by a radiologist (NB) blinded to all of the clinical information, and a score (Bhalla score ${ }^{15}$ ) was calculated on the basis of severity and/or extent of nine morphological changes: a score of $0-3(0=$ best, $3=$ worst $)$ was assigned for the severity of bronchiectasis, peribronchial thickening, the extent of brochiectasis, the extent of mucus plugging, abscesses or sacculations, and the generations of the bronchial divisions involved; a score of $0-2(0=$ best, 2 =worst) was assigned for emphysema and collapse or consolidation. The highest possible score was 25 .

Patients who were able to cooperate underwent pulmonary function testing within two weeks of the chest HRCT using a water filled spirometer (Biomedin, Padua, Italy). The best forced expiratory volume in one second $\left(\mathrm{FEV}_{\mathrm{l}}\right)$ and vital capacity (VC) of three attempts were recorded at baseline and after two inhalation of salbutamol via metered dose inhalers, and the $\mathrm{FEV}_{1} / \mathrm{VC}$ ratio was computed.

Sputum was obtained in patients free from an acute respiratory illness and who were able to cooperate; bacterial cultures were done according to conventional routine procedures.

Chronic sinusitis was diagnosed clinically in the presence of at least two of the following symptoms for at least three months: mucous or purulent nasal discharge, nasal congestion, and posterior nasal drainage. ${ }^{16}{ }^{17}$ The symptoms were separately scored for severity on a scale of 0 (none) to 3 (severe) $:^{18}$ a positive diagnosis required a minimum score of 4. The nasal cavities were direct visualised by means of a $2.4 \mathrm{~mm}$ Pentax FNL-7RP2 flexible fibreoptic endoscope (Asahi Optical Co., Tokyo, Japan) in order to confirm sinus infection (purulent material emanating from the sinus drainage region) and identify polyps or structural abnormalities. ${ }^{19}$

\section{Follow up study}

Sixteen patients attending the Department of Paediatrics of the University of Milan (six with XLA, one with CVID, and nine with IgA-D) participated in the prospective three year follow up study. All received intensive chest physiotherapy. Acute lower respiratory tract infections were treated empirically or on the results of bacterial cultures of sputum with intravenous or oral antibiotics. None of the patients received prophylactic antibiotic therapy. Questionnaires concerning respiratory symptoms were completed every 1-3 months. HRCT examinations were repeated at the end of the follow up in 11 patients with an HRCT score $\geqslant 4$ at the first examination. Changes in HRCT score (score at follow up score at entry) and difference in the number of months/year of productive cough over the follow up and in the previous two years (clinical score) were calculated for each patient.

Lung function tests were repeated at intervals of three to six months and at the end of follow up; $\mathrm{FEV}_{1}$ was used to assess deterioration over time.

The patients with chronic sinusitis were treated with amoxicillin-potassium clavulanate $(50 \mathrm{mg} / \mathrm{kg} / \mathrm{day})$ and clindamycin $(10-20 \mathrm{mg} / \mathrm{kg} /$ day $)$ for two weeks. All of the patients were treated with saline nasal washes and intranasal beclomethasone (one puff in each nostril three times a day) for four weeks. The patients with nasal polyposis also received a three week course of oral prednisone ( $1 \mathrm{mg} / \mathrm{kg}$ / day). Three to four weeks after the completion of treatment, the patients were clinically re-evaluated for the presence of sinusitis. If the symptoms had resolved or improved (score $<4)$, the response to treatment was confirmed by flexible nasal endoscopy; if they had not resolved (score $\geqslant 4$ ), a second course of treatment was prescribed and flexible nasal endoscopy was performed after the second antibiotic course. The children in whom medical management failed underwent a coronal HRCT scan in order to evaluate any abnormalities before treatment (endoscopic sinus surgery).

\section{Statistical methods}

HRCT scores were compared between groups using the Mann-Whitney test.

The relation between variables was assessed with parametric or distribution-free methods as appropriate.

In analysing the relation of clinical to HRCT score, that was not the same over the range of score values, a piecewise linear function was used (procedure MKspline of the STATA 7 statistical package, College Station, Texas, USA). This is a function composed of linear segments, each limited to an interval of $\mathrm{x}$ values, that are constrained to join each other at the connection points ("knots"). A standard regression could 
then be used, because the distribution of clinical scores, conditional on HRCT score, was normal.

\section{RESULTS}

\section{Evaluation of pulmonary and sinus disease at entry}

Lung function tests were performed at entry in the 21 patients who were able to cooperate and were normal in 17. Four patients (patients 3, 7, 12, and 14 in table 1) had a mild obstructive syndrome, defined as $\mathrm{FEV}_{1} / \mathrm{FVC}$ of $<80 \%$ and $>65 \%$ of the theoretical value ${ }^{20}$ with poorly reversible airflow limitation (improvement in $\mathrm{FEV}_{1}<10 \%$ after salbutamol inhalation). Table 1 shows the HRCT scan scores of each patient at entry. Six patients (two with XLA and four with IgA-D) had a completely normal HRCT scan (score 0); the scores of the other patients varied widely from 1 to 15 . The most frequent abnormalities were those relating to airway diseases (bronchiectasis, peribronchial thickening). Mildly or moderately severe bronchiectasis (scorel-2) was present in 17 patients, being predominant in the lower and middle lobes, and sparing the upper lobes. In the same patients peribronchial thickening of equivalent severity was also found. Mucus plugging involving 1-5 segments (score 1) or 6-9 segments (score 2) was present in five patients. None of the patients had abscesses or bronchiectatic sacculations. Segmental or subsegmental collapse or consolidation and mild emphysema (score 1) were found in, respectively, nine and three patients.

The patients with XLA, ARA, or CVDI tended to have higher HRCT scores (median 7, range 0-15) than those with IgA-D (median 4, range $0-12)(p=0.075$, Mann-Whitney test), but the scores varied widely.

Figure 1 shows the relation between the number of months with productive cough/year in the two years before entry and the severity of HRCT score. A good correlation $(p=0.0001)$ was found only for patients with a Bhalla score $\geqslant 4$. No correlation was found between the HRCT score at entry and lifetime pneumonia episodes $(r=0.22)$.

Data on bacterial cultures of sputum were available for 18 patients who were able to cooperate. Bacterial growth was observed in sputum samples of 11 patients when concentrations of $\geqslant 10^{5} \mathrm{cfu} / \mathrm{ml}$ were used as a cut off point. Haemophilus influenzae was detected in nine samples and Streptococcus pneumoniae in two.

Thirteen patients (six with agammaglobulinaemia, one with CVID, and six with IgA-D) had chronic sinusitis at entry, four of whom had concomitant nasal polypoid disease (table 1).

\section{Follow up study}

Eleven of the 16 patients who were followed up (six with XLA, one with CVID, and four with IgA-D) repeated a HRCT scan after two to three years; the HRCT score increased in two patients, decreased in six (in particular for mucus plugging and peribronchial thickening) and remained stable in three (fig 2). In the case of these 11 patients, there was a correlation between the difference in months of productive cough/year (clinical score) during follow up and in the two years before entry and the difference in HRCT score $(r=0.6$; $\mathrm{p}=0.05$ ) (fig 2). Lung function testing showed deterioration during the follow up in only one patient (patient 3 in table 1) who had a difference in HRCT score of $+2 ; \mathrm{FEV}_{1}$ decreased from $87 \%$ of predicted to $67 \%$. Eight of the ten patients with chronic sinusitis who entered the follow up were successfully treated with medical therapy, including two with polypoid degeneration of the middle turbinate. In two patients with XLA, sinusitis recurred after two months; further follow up of these patients was impossible due to lack of compliance. Endoscopic sinus surgery led to a clinical improvement over a three year period in the other two patients who had massive nasal polyposis.

\begin{tabular}{|c|c|c|c|c|c|c|}
\hline Patients & $\begin{array}{l}\text { Age at diagnosis } \\
\text { (y) }\end{array}$ & $\begin{array}{l}\text { Age at entry } \\
\text { (y) }\end{array}$ & $\begin{array}{l}\text { Cough and phlegm: } \\
\text { past } 2 \text { y }(\mathrm{mth} / \mathrm{y})\end{array}$ & $\begin{array}{l}\text { Lifetime } \\
\text { pneumonia } \\
\text { (no.) }\end{array}$ & $\begin{array}{l}\text { HRCT } \\
\text { score }\end{array}$ & Sinus disease \\
\hline \multicolumn{7}{|l|}{ XLA } \\
\hline 1 & 6.0 & 6.0 & 10 & 3 & 15 & CS \\
\hline 2 & 3.0 & 14.7 & 8 & 4 & 13 & \\
\hline 3 & 5.7 & 14.3 & 12 & 3 & 12 & CS+MNP \\
\hline 4 & 12.0 & 19.3 & 3 & 9 & 9 & \\
\hline 5 & 15.0 & 15.5 & 7 & 4 & 8 & \\
\hline 6 & 7.0 & 14.4 & 4 & 8 & 7 & \\
\hline 7 & 1.5 & 19.2 & 3 & 5 & 6 & \\
\hline 8 & 0.6 & 15.0 & 4 & 0 & 4 & \\
\hline 9 & 0.8 & 11.2 & 5 & 2 & 1 & CS \\
\hline 10 & 2.4 & 8.3 & 4 & 0 & 0 & CS \\
\hline \multirow{2}{*}{\multicolumn{7}{|c|}{ ARA }} \\
\hline & & & & & & \\
\hline 12 & 4.0 & 16.3 & 10 & 18 & 10 & CS \\
\hline 13 & 1.0 & 13.5 & 6 & 13 & 6 & CS \\
\hline \multicolumn{7}{|l|}{ CVID } \\
\hline 14 & 3.0 & 19.8 & 6 & 3 & 11 & CS \\
\hline 15 & 3.5 & 6.8 & 4 & 1 & 4 & \\
\hline \multicolumn{7}{|l|}{$\lg A-D$} \\
\hline 16 & 4.0 & 8.7 & 8 & 1 & 12 & \\
\hline 17 & 6.0 & 10.7 & 6 & 1 & 7 & CS+MNP \\
\hline 18 & 3.0 & 5.6 & 6 & 3 & 6 & CS \\
\hline 19 & 1.7 & 2.9 & 6 & 3 & 4 & CS \\
\hline 20 & 12.7 & 12.7 & 3 & 3 & 4 & $C S+N P D$ \\
\hline 21 & 2.3 & 2.7 & 4 & 1 & 0 & \\
\hline 22 & 4.3 & 4.9 & 4 & 2 & 0 & CS \\
\hline 23 & 3.0 & 13.2 & 6 & 1 & 0 & $C S+N P D$ \\
\hline 24 & 3.1 & 8.7 & 3 & 1 & 0 & \\
\hline
\end{tabular}

$\mathrm{XLA}, \mathrm{X}$ linked agammaglobulinaemia; ARA, autosomal recessive agammaglobulinaemia; CVID, common variable immunodeficiency; IgA-D, IgA deficiency; CS, chronic sinusitis; MNP, massive nasal polyposis; NPD, nasal polypoid degeneration of the middle turbinates. 


\section{DISCUSSION}

Early diagnosis, appropriate immunoglobulin replacement therapy, intensive chest therapy, and antibiotics have changed the clinical history of patients with humoral immunodeficiency, ${ }^{2321}$ but many still present with chronic productive cough, the hallmark of chronic bronchitis or sinusitis. ${ }^{1622}$ An objective assessment of the extent of lung and sinus disease in patients with chronic respiratory symptoms and a standardised therapeutic approach and follow up are highly important to ensure long term survival without major complications.

We found that pulmonary abnormalities, chronic sinusitis, or both developed in the majority of our patients with humoral deficiency and productive cough for at least three months/year. HRCT proved to be a sensitive means of imaging morphological changes in $75 \%$ of the patients, including five with agammaglobulinaemia and no history of lower airways infection at diagnosis (data not shown) and five of nine patients with IgA-D. The most frequent abnormalities were mild and moderate bronchiectasis and peribronchial thickening. In a recent retrospective study of children with agammaglobulinaemia, Quartier and colleagues $^{2}$ documented bronchiectasis on CT scan in only $26 \%$ of their patients; however, some did not have chronic respiratory symptoms, and the authors did not perform HRCT scans.

HRCT has been considered the most reliable non-invasive method of assessing bronchiectasis in adults and children with primary hypogammaglobulinaemia. ${ }^{12} 1323$ The scoring system we used was originally devised for children with cystic fibrosis affected by a more severe lung disease, ${ }^{15}$ but it has been subsequently and successfully applied to describe the extent of lung disease in children with antibody deficiency disorders. ${ }^{12}$ The detection and definition of morphological changes in the airways of most of our patients by means of this score encouraged us to pursue chest physiotherapy and aggressive antibiotic therapy during acute airway infections. Mucus plugging, which is crucial in the pathogenesis of bronchiectasis, and peribronchial thickening, which reflects the presence of chronic infection resulting in inflammation, are amenable to medical management as shown by their improvement in some of our patients during follow up.

HRCT revealed progression of bronchiectasis despite adequate immunoglobulin replacement therapy has been reported in very few children with humoral immunodeficiency. ${ }^{12} 13$ We found that the pulmonary lesions in our patients with a Bhalla score $\geqslant 4$ and a good level of compliance not only to medical therapy but also to intensive chest physiotherapy did not significantly progress over a

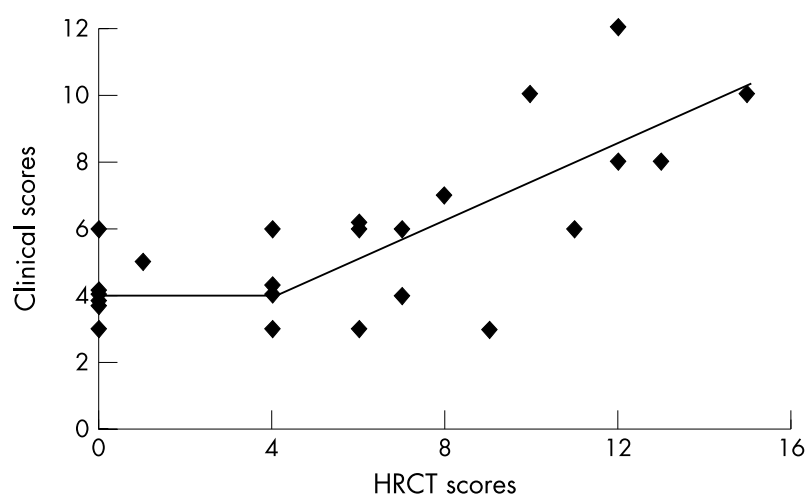

Figure 1 Relation between HRCT scores at entry and the number of months/year of productive cough over the previous two years (clinical score). $R^{2}=0.58$.

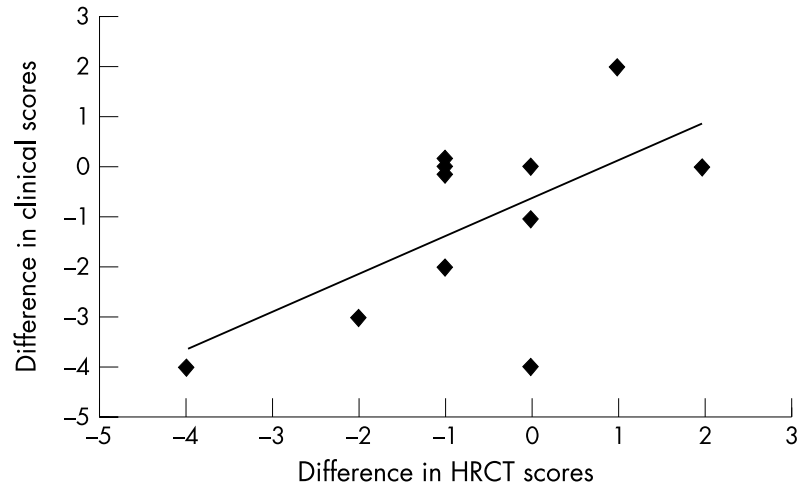

Figure 2 Correlation between difference in HRCT scores (score at follow up - score at entry) and difference in number of months/year of productive cough (clinical score) over the follow up and the two years before entry. $r=0.60$.

three year period, but the small number of patients followed up in this and previous studies ${ }^{12}{ }^{13}$ precludes any definite conclusions. In these patients we found a fairly good correlation between HRCT scores and clinical findings (months of productive cough/year); we therefore would not suggest subsequent annual or bi-annual HRCT scans unless their clinical status or lung function tests deteriorate. Reducing radiation exposure should also be a consideration, as recently discussed in several studies: $:^{25}$ even if CT parameters are adjusted for age to minimise the radiation dose, the lifetime cancer risk per unit of radiation is greater for children-who face a large lifetime background risk of cancer mortality-than adults. ${ }^{24}$ In patients in whom no damage or only very mild damage can be shown at HRCT scan, it is important to evaluate whether chronic productive cough may be due to the presence of chronic sinusitis with a posterior nasal drainage, as occurred in four of our seven patients with an HRCT score of $0-1$ (table 1). This could explain at least in part the lack of relation between HRCT score and clinical score in these patients. As expected, the results of the pulmonary function tests showed an obstructive pattern at entry only in four patients with more severe disease. This is in agreement with the results of the study of Quartier and colleagues ${ }^{2}$ in paediatric patients with agammaglobulinaemia treated with high dose intravenous immunoglobulins, whereas, in a previous study, an obstructive or restrictive pattern was found in half of a series of older patients with hypogammaglobulinaemia who received intramuscular gammaglobulins. ${ }^{26}$

Chronic sinusitis continues to be a problem in patients with humoral immunodeficiency. The high prevalence of the disease in our patients with hypogammaglobulinaemia (7/15) is similar to that reported in patients with XLA and CVDI treated with intramuscular or intravenous immunoglobulins. ${ }^{245}$ We also diagnosed chronic sinusitis in 6/9 patients with IgA-D and chronic cough and phlegm. Diagnosing chronic sinusitis thoroughly and defining a therapeutic regimen to control the disease is therefore of great importance. According to a recently published "Consensus on the management of sinusitis" in children, ${ }^{27}$ we decided to rely mainly on clinical criteria to diagnose and then to follow chronic sinusitis. Nasal flexible endoscopy was easy to tolerate by the patients and particularly helpful in supporting the clinical diagnoses, identifying early polypoid disease, and objectively assessing treatment response. The management of sinusitis in patients with humoral immunodeficiency is controversial, ${ }^{28}$ and given the rarity of the condition, it is not surprising that no randomised clinical trial results are 
available. We decided to adopt a combined therapeutic approach (antibiotics + intranasal/oral steroids + saline nasal washes) as suggested for non-immunodeficient patients. ${ }^{16}$ With regard to the choice of antibiotic (amoxicillin-clavulanate and clindamycin) and the duration of therapy, we considered that the causative agents of airway infections in patients with chronic sinusitis are usually common respiratory pathogens such as Streptococcus pneumoniae, Haemophilus influenzae, and Moraxella catarrhalis, that anaerobes may play a role, and that most of the patients had already received in the previous months antibiotics that may have selected resistant strains. ${ }^{16}{ }^{17}$ Although a formal assessment of this treatment option is beyond the scope of the present study, the results obtained are interesting because, unlike previous authors, ${ }^{8}$ we found that maximal medical therapy and, for patients with massive polyposis, endoscopic sinus surgery were effective in the majority of cases.

In conclusion, in order to ensure the optimal treatment of patients with humoral immunodeficiency and chronic respiratory symptoms, an effort has to be made to ascertain the presence of chronic sinusitis and carefully delineate the extent of lung damage by means of an objective and sensitive method such as HRCT. The criterion we used of investigating only the patients with chronic cough and phlegm may be particularly useful in discriminating which patients with IgA-D need a closer follow up. Prospective, collaborative, and randomised clinical studies are needed in order to provide more information concerning the optimal treatment of chronic sinusitis and the long term progression of pulmonary disease in different humoral deficiency disorders. On the basis of this limited experience, chronic sinusitis can be effectively treated in most patients by means of maximal medical therapy (one or two courses of a combination of broad spectrum antimicrobials, anti-inflammatory agents, and saline nasal washes), and, in cases with massive nasal polyposis, endoscopic sinus surgery. In optimally treated and clinically stable patients, if a definite lung damage is shown at HRCT, the close correlation between HRCT scores and clinical findings, and the favourable outcome of pulmonary disease, suggest that chest HRCT scan should not be performed annually as previously advocated..$^{12}{ }^{13}$ It remains to be clarified how often HRCT should be repeated in order to reveal whether silent progression of pulmonary lesions may occur despite appropriate therapy.

\section{ACKNOWLEDGEMENTS}

We thank Dr Viviana Moschese and Dr Luigi Gagliardi for helpful comments on the manuscript and Dr Kevin Smart for revision of the text.

\footnotetext{
Authors' affiliations

F Rusconi, C Panisi, R M Dellepiane, M C Pietrogrande, Department of

Paediatrics, University of Milan, ICP, Milan, Italy

G Felisati, Department of Otolaryngology, University of Milan, ICP, Milan, Italy

F Cardinale, B Martire, Department of Paediatric Medicine, University of Bari, Bari, Italy

L Chini, Department of Paediatrics, Università Tor Vergata, Rome, Italy N Bonelli, Department of Paediatric Radiology, ICP Milan, Italy

Supported by MURST (Ministero dell'Università e della Ricerca Scientifica e Tecnologica), Cofinanziamento 1997
}

Presented in abstract form at the annual meeting of the American Thoracic Society, May 2002, Atlanta, USA

\section{REFERENCES}

1 Roifman CM, Gelfand EW. Replacement therapy with high dose intravenous gammaglobulins improves chronic pulmonary disease in patients with hypogammaglobulinemia. Pediatr Infect Dis J 1988;7:S92-6.

2 Quartier $\mathbf{P}$, Debrè $M$, De Blic J, et al. Early and prolonged intravenous immunoglobulin therapy in childhood agammaglobulinemia: a retrospective survey of 31 patients. J Pediatr 1999;134:589-96.

3 Eijkhout HE, van der Meer JWS, Kallenberg CGM, et al. The effect of two different dosages of intravenous immunoglobulin on the incidence of recurrent infections in patients with primary hypogammaglobulinemia. A randomized, double-blind, multicenter crossover trial. Ann Intern Med 2001;135:165-74.

4 Hausser C, Virelizier JL, Buriot D, et al. Common variable

hypogammaglobulinemia in children. Am J Dis Child 1983;137:833-7.

5 Lederman HM, Winkelstein JA. X-linked agammaglobulinemia: an analysis of 96 patients. Medicine 1985;64:145-56.

6 Watts WJ, Watts MB, Dai W, et al. Respiratory dysfunction in patients with common variable hypogammaglobulinemia. Am Rev Respir Dis 1986;134:699-703.

7 Dukes RJ, Rosenow EC III, Hermans PE. Pulmonary manifestations of hypogammaglobulinaemia. Thorax 1978;33:603-7.

8 Buehring I, Friedrich B, Schaaf J, et al. Chronic sinusitis refractory to standard management in patients with humoral immunodeficiencies. Clin Exp Immunol 1997; 109:468-73.

9 Holinski-Feder $E$, Weiss $M$, Brandau $O$, et al. Mutation screening of the BTK gene in 56 families with X-linked agammaglobulinemia (XLA): 47 unique mutations without correlation to clinical course. Pediatrics 1998; 101:276-84.

10 Kornfeld SJ, Haire RN, Strong SJ, et al. Extreme variation in X-linked agammaglobulinemia phenotype in a three-generation family. J Allergy Clin Immunol 1997; 100:702-6.

11 Hammarström L, Vorechovsky I, Webster D. Selective IgA deficiency (SigAD) and common variable immunodeficiency. Clin Exp Immunol 2000;120:225-31.

12 Manson D, Reid B, Dalal I, et al. Clinical utility of high-resolution pulmonary computed tomography in children with antibody deficiency disorders. Pediatr Radiol 1997;27:794-8.

13 Kainulainen L, Varpula M, Liippo K, et al. Pulmonary abnormalities in patients with primary hypogammaglobulinemia. J Allergy Clin Immunol 1999; 104:1031-6.

14 Conley ME, Notarangelo LD, Etzioni A. Diagnostic criteria for primary immunodeficiencies. Clin Immunol 1999:93:190-7.

15 Bhalla M, Turcios N, Aponte V, et al. Cystic fibrosis: scoring system with thinsection CT. Radiology 1991;179:783-8.

16 Wald ER. Chronic sinusitis in children. J Pediatr 1995; 127:339-47.

17 Spector SL, Bernstein IL, Li JT, et al. Parameters for the diagnosis and management of sinusitis. J Allergy Clin Immunol 1998;102:S107-44.

18 Nguyen KL, Corbett ML, Garcia DP, et al. Chronic sinusitis among pediatric patients with chronic respiratory complaints. J Allergy Clin Immunol 1993;92:824-30.

19 Castellanos J, Axelrod D. Flexible fiberoptic rhynoscopy in the diagnosis of sinusitis. J Allergy Clin Immunol 1989;83:91-4.

20 Knudson RJ, Lebowitz MD, Holberg CJ, et al. Changes in the normal maximal expiratory flow-volume curve with growth and aging. Am Rev Respir Dis 1983; 127:725-34.

21 Ochs HD, Smith CIE. X-linked agammaglobulinemia, a clinical and molecular analysis. Medicine 1996;75:287-99.

22 Morgan WJ, Taussig LM. The chronic bronchitis complex in children. Pediatr Clin North Am 1984;31:851-64.

23 Obregon RG, Lynch DA, Kaske T, et al. Radiologic findings of adult primary immunodeficiency disorders. Chest 1994;106:490-5.

24 Brenner DJ, Elliston CD, Hall EJ, et al. Estimated risk of radiation-induced fatal cancer from pediatric CT. AJR 2001; 176:289-96.

25 Donnelly LF, Emery KH, Brody, et al. Minimizing radiation dose for pediatric body applications of single-detector helical CT: strategies in a large children's hospital. AJR 2001;176:303-6.

26 Sweinberg SK, Wodell RA, Grodofsky MP, et al. Retrospective analysis of the incidence of pulmonary disease in hypogammaglobulinemia. J Allergy Clin Immunol 1991;88:96-104.

27 Clement PAR, Bluestone CD, Gordts F, et al. Management of rhinosinusitis in children. Arch Otolaryngol Head Neck Surg 1998;124:31-4.

28 Lusk RP, Polmar SH, Muntz HR. Endoscopic ethmoidectomy and maxillary antrostomy in immunodeficient patients. Arch Otolaryngol Head Neck Surg $1991 ; 117: 60-3$. 